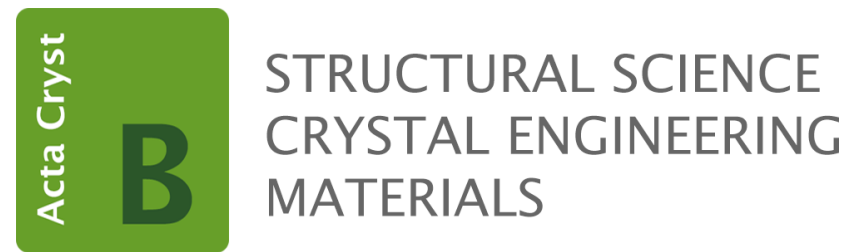

Volume 71 (2015)

Supporting information for article:

A new polymorph of 1-hydroxy-2-naphthoic acid obtained during failed co-crystallization experiments

Qi Zhang, Meiqi Li and Xuefeng Mei 


\section{S1. Experimental}

\section{S1.1. Materials}

HNA raw material is purchased from Aladdin Reagent Company Limited and all analytical grade solvents were purchased from Sinopharm Chemical Reagent Company Limited, and used without further purification.

\section{S1.2. Preparation of HNA Polymorphs}

HNA form I and form II were prepared by slow evaporation at room temperature from diethyl ether and acetonitrile, repectively.

\section{S1.3. Thermogravimetric Analysis (TGA)}

Thermogravimetric analysis was carried out in a Netzsch TG209 F3 equipment, using $\mathrm{N}_{2}$ as dry air with a flow of $20 \mathrm{~mL} / \mathrm{min}$ and a scan rate of $10{ }^{\circ} \mathrm{C} / \mathrm{min}$.

\section{S1.4. Differential Scanning Calorimetry (DSC)}

Differential scanning calorimetry was performed with a PerkinElmer DSC 8500 instrument. Samples weighting 3-5 mg were heated in standard aluminum pans at scan rates from $2{ }^{\circ} \mathrm{C} / \mathrm{min}$ to $80^{\circ} \mathrm{C} / \mathrm{min}$ under nitrogen gas flow of $20 \mathrm{~mL} / \mathrm{min}$. Two-point calibration using indium and tin was carried out to check the temperature axis and heat flow of the equipment.

\section{S1.5. Single crystal X-ray diffraction}

Single crystal X-ray diffraction measurements for were conducted on a Bruker Smart Apex II diffractometer using Mo-K $\alpha$ radiation $(\lambda=0.71073 \AA)$ with a graphite monochromator. Integration and scaling of intensity data was accomplished using the SAINT program (Bruker, 2000). The structures were solved by Direct Methods using SHELXS97 (Sheldrick, 2008) and refinement was carried out by full-matrix least-squares technique using SHELXL97 (Sheldrick, 2008). The heavy atoms were refined anisotropically. The O-H hydrogens were located from difference electron density maps and their coordinates were allowed to refine 
freely while their displacement parameters were set to Uiso $(H)=1.5$ Ueq $(O)$. The $C-H$ hydrogens were placed in calculated positions and refined using a riding model with $\mathrm{C}-\mathrm{H}=$ $0.93 \AA$ and with Uiso $(\mathrm{H})=1.2 \mathrm{Ueq}(\mathrm{C})$.

\section{S1.6. Powder X-ray Diffraction}

PXRD were measured on a Bruker D8-ADVANCE X-ray diffractometer using a $\mathrm{Cu}-\mathrm{K} \alpha$ radiation $(\lambda=1.5418 \AA)$. The voltage and current were $40 \mathrm{kV}$ and $40 \mathrm{~mA}$, respectively. Samples were measured in reflection mode in the $2 \theta$ range $3-40^{\circ}$ with a scan speed of 15 \% $/$ min (step size $0.025^{\circ}$, step time $0.1 \mathrm{~s}$ ) using a LynxEye detector. All data were acquired at ambient temperature. Data were imaged and integrated with RINT Rapid and peak-analyzed with Jade 6.0 software from Rigaku. Calibration of the instrument was performed using Corindon (Bruker AXS Korundprobe) standard.

Variable-temperature X-ray powder diffraction patterns were recorded with a XPH-300 hot stage (Changfang, China) attached to the Bruker D8 Advance diffractometer (Bruker, Germany). A heating rate of $10{ }^{\circ} \mathrm{C} / \mathrm{min}$ was used during all of these determinations. Approximately $50 \mathrm{mg}$ samples were weighed into the sample holder, taking care not to introduce a preferential orientation of crystals.

\section{S1.7. Fourier-transform Infrared (FTIR)}

Fourier-transform Infrared (FTIR) spectra were collected by a Nicolet-Magna FT-IR 750 spectrometer in the range of 4000 to $400 \mathrm{~cm}^{-1}$ with a resolution of $4 \mathrm{~cm}^{-1}$ at ambient conditions.

\section{S1.8. Hot-Stage Microscopy (HSM)}

All HSM examinations were performed on a XPV-400E Polarizing microscope and a XPH-300 hot stage coupled with a JVC TK-C9201 EC digital video recorder (Shanghai Changfang Optical Instrument Company). 
Table S1 Crystallographic data for HNA polymorphs

For all structures: $\mathrm{C}_{11} \mathrm{H}_{8} \mathrm{O}_{3}, M_{\mathrm{r}}=188.17$. Experiments were carried out at $296 \mathrm{~K}$ with Mo $K \alpha$ radiation.

$\mathrm{H}$ atoms were treated by a mixture of independent and constrained refinement.

\begin{tabular}{|c|c|c|}
\hline & mo_I & mo_II \\
\hline \multicolumn{3}{|l|}{ Crystal data } \\
\hline $\begin{array}{l}\text { Crystal system, space } \\
\text { group }\end{array}$ & Monoclinic, $P 2_{1} / n$ & Monoclinic, $C 2 / c$ \\
\hline$a, b, c(\AA)$ & $6.9798(9), 3.8107$ (6), $32.790(5)$ & 20.920 (5), $6.9926(14), 14.688$ (3) \\
\hline$\beta\left(^{\circ}\right)$ & $93.901(8)$ & $125.339(10)$ \\
\hline$V\left(\AA^{3}\right)$ & $870.1(2)$ & $1752.7(6)$ \\
\hline$Z$ & 4 & 8 \\
\hline$\mu\left(\mathrm{mm}^{-1}\right)$ & 0.11 & 0.11 \\
\hline Crystal size (mm) & $0.44 \times 0.32 \times 0.11$ & $0.42 \times 0.34 \times 0.11$ \\
\hline \multicolumn{3}{|l|}{ Data collection } \\
\hline Absorption correction & - & - \\
\hline $\begin{array}{l}\text { No. of measured, } \\
\text { independent and } \\
\text { observed }[I>2 \sigma(I)] \\
\text { reflections }\end{array}$ & $6187,1491,1020$ & $7678,2015,1239$ \\
\hline$R_{\text {int }}$ & 0.038 & 0.034 \\
\hline$(\sin \theta / \lambda)_{\max }\left(\AA^{-1}\right)$ & 0.594 & 0.651 \\
\hline Refinement & & \\
\hline
\end{tabular}




\begin{tabular}{|l|l|l|}
\hline$R\left[F^{2}>2 \sigma\left(F^{2}\right)\right], w R\left(F^{2}\right)$, & $0.038,0.097,0.96$ & $0.068,0.226,1.04$ \\
$S$ & & \\
\hline No. of reflections & 1491 & 2015 \\
\hline$\left.\Delta\rangle_{\max }, \Delta\right\rangle_{\min }\left(\mathrm{e} \AA^{-3}\right)$ & $0.15,-0.16$ & $0.49,-0.27$ \\
\hline
\end{tabular}

* Computer programs: SHELXS97 (Sheldrick, 2008), SHELXL97 (Sheldrick, 2008).

The crystallographic data of forms I and II (CCDC 1019893-1019894) is also available through Cambridge Crystallographic Data Centre 
Table S2 Hydrogen bond parameters and short $\mathrm{O} \cdots \mathrm{O}$ contacts

\begin{tabular}{lll}
\hline Polymorphs & Form I & Form II \\
\hline$(\mathrm{O} 3-\mathrm{H} 2) / \AA$ & $0.93(2)$ & $0.93(4)$ \\
$\left(\mathrm{H} 2 \cdots \mathrm{O} 22^{\mathrm{i}}\right) / \AA$ & $1.72(2)$ & $1.74(4)$ \\
$\left(\mathrm{O} 3 \cdots \mathrm{O} 22^{\mathrm{i}}\right) / \AA$ & $2.650(2)$ & $2.650(2)$ \\
symmetry operation for (i) & $-\mathrm{x}+1,-\mathrm{y}+2,-\mathrm{z}+2$ & $-\mathrm{x}+1, \mathrm{y},-\mathrm{z}+0.5$ \\
$(\mathrm{O} 1-\mathrm{H} 1) / \AA$ & $0.92(2)$ & $0.86(4)$ \\
$(\mathrm{H} 1 \cdots \mathrm{O} 2) / \AA$ & $1.77(2)$ & $1.83(3)$ \\
$(\mathrm{O} 1 \cdots \mathrm{O} 2) / \AA$ & $2.600(2)$ & $2.616(2)$ \\
$\left(\mathrm{O} 1 \cdots \mathrm{O} 11^{\mathrm{ii}}\right) / \AA$ & - & $2.979(2)$ \\
$\mathrm{Symmetry}$ operation for (ii) & - & $-\mathrm{x}+1.5,-\mathrm{y}+0.5,-\mathrm{z}+1$ \\
\hline
\end{tabular}

Table S3 Table S3. Columnar stacking parameters

\begin{tabular}{lll}
\hline Polymorphs & Form I & Form II \\
\hline Centroid* to centroid distance $(\AA)$ & $3.811(2)$ & $3.849(3)$ \\
Centroid to plane distance $(\AA)$ & $3.474(2)$ & $3.536(3) ; 3.451(3)$ \\
Interplanar angle $\left(^{\circ}\right)$ & 0 & 4.7 \\
Angle between b-direction and the axis of the & 0 & $24.7(1)$ \\
stack $\left(^{\circ}\right)$ & & \\
Symmetry operations & $\mathrm{x}, \mathrm{y}+1, \mathrm{z}$ & $-\mathrm{x}+1.5, \mathrm{y}-0.5,-\mathrm{z}+0.5$ \\
& & $-\mathrm{x}+1.5, \mathrm{y}+0.5,-\mathrm{z}+0.5$ \\
\hline
\end{tabular}

* The centroids were calculated by atoms C1, C2, C3, C4, C5 and C10. 

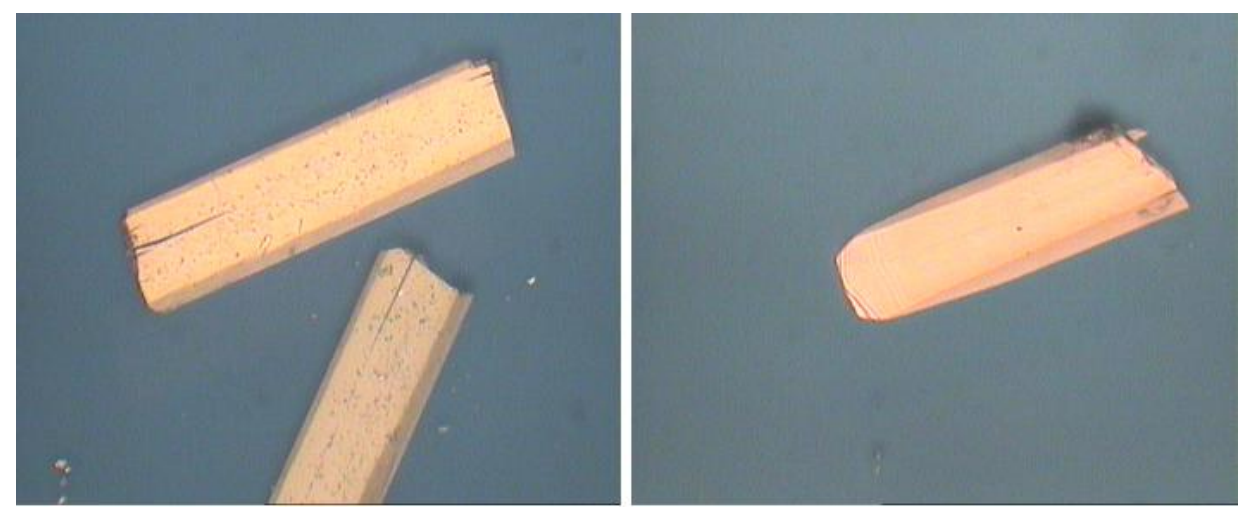

Figure S1 HSM photographs of HNA Form I (left) and Form II (right).
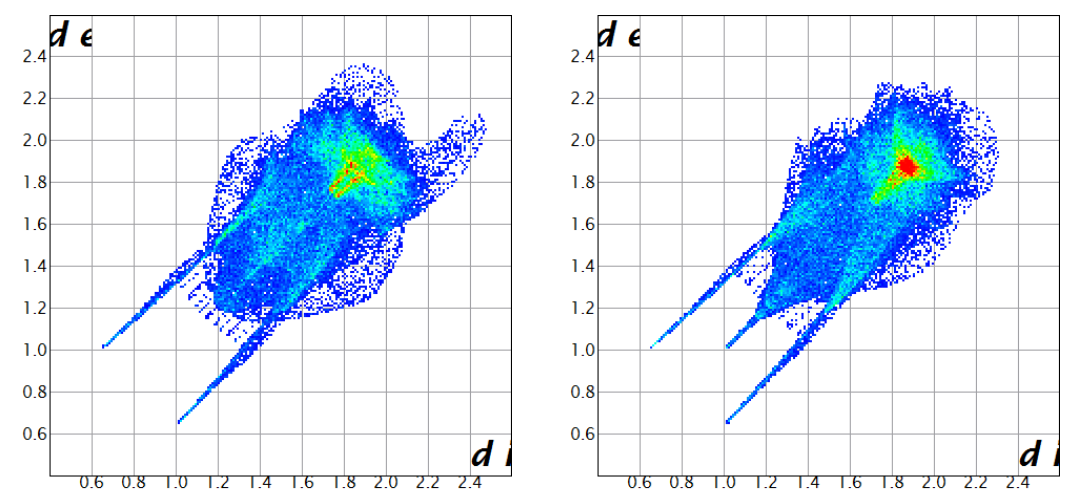

Figure S2 2D fingerprint plots of HNA Form I (left) and Form II (right).

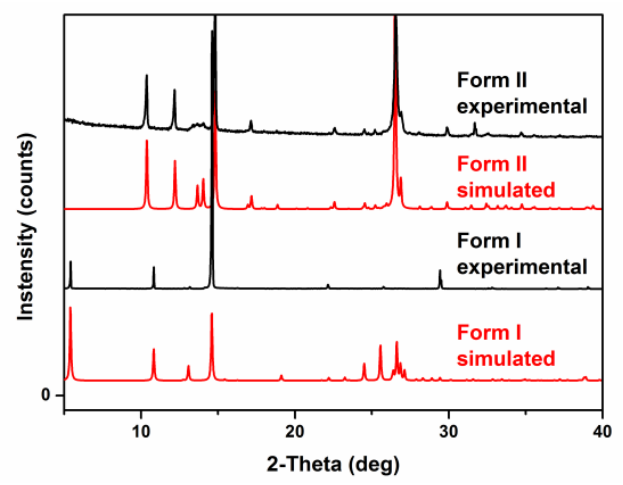

Figure S3 Compared PXRD patterns of HNA polymorphs. 


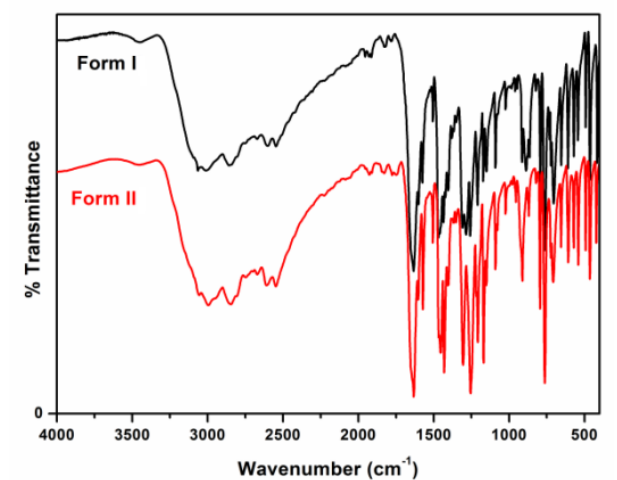

(a)

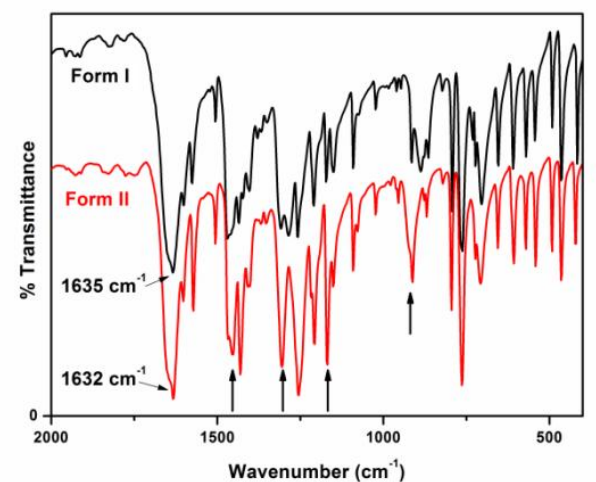

(b)

Figure S4 Compared FT-IR spectra of HNA polymorphs (a) full range, (b) $2000-400 \mathrm{~cm}^{-1}$.

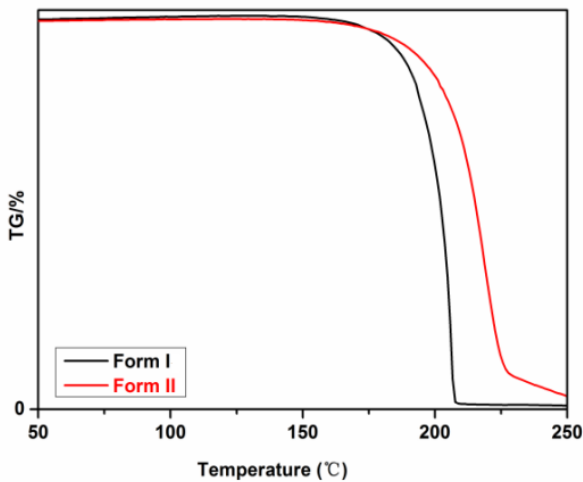

(a)

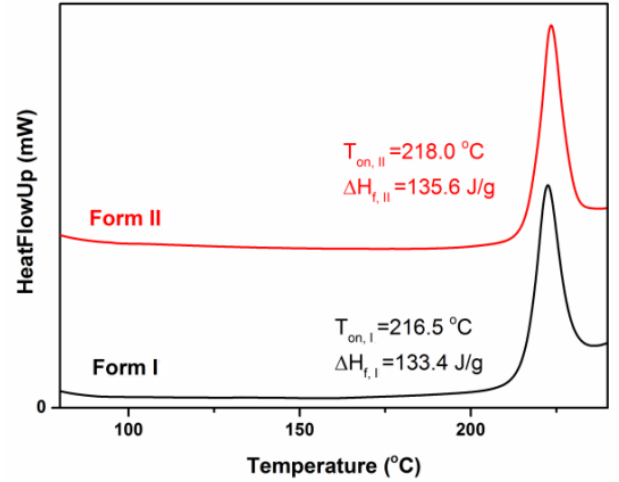

(b)

Figure S5 Compared TGA (a) and DSC (b) thermograms of HNA polymorphs.

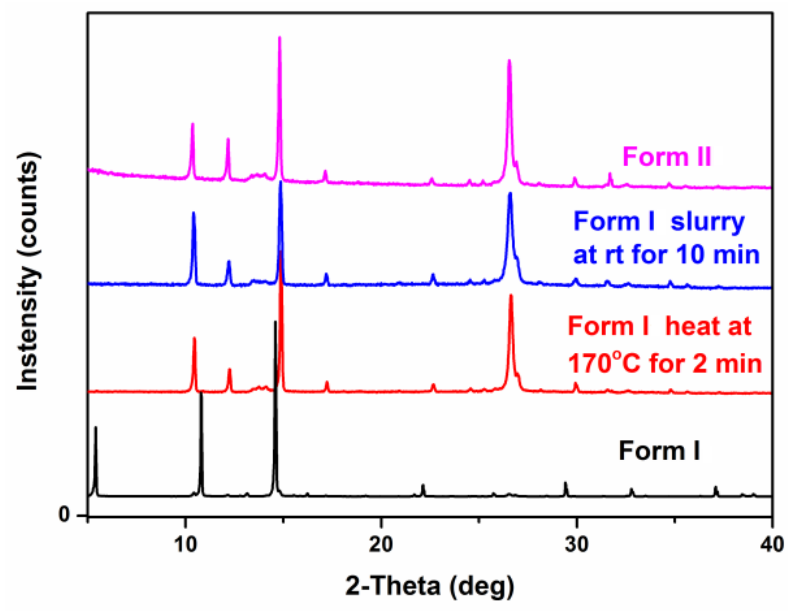

Figure S6 Compared PXRD patterns for HNA form transformations. 


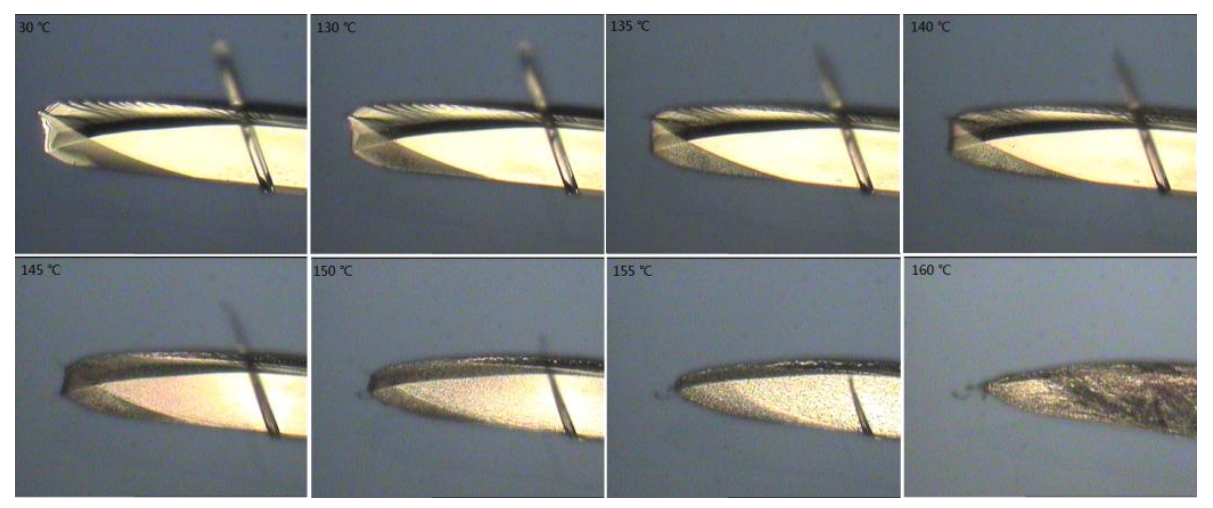

Figure S7 HSM photographs of HNA Form I heated at different temperatures.

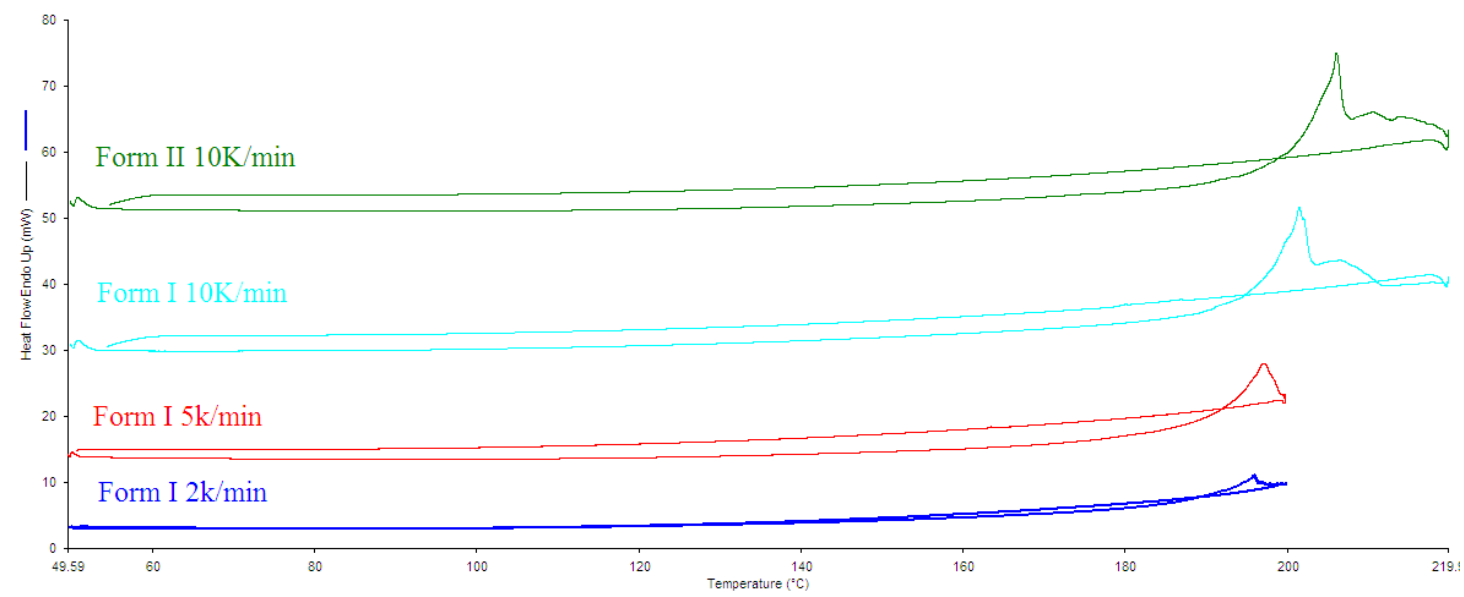

Figure S8 DSC thermograms of HNA polymorphs using lower heating/cooling-rate. 